\title{
Primary polypseudophakia for cataract surgery in hypermetropic eyes: refractive results and long term stability of the implants within the capsular bag
} Haralabos Eleftheriadis, Andrea Sciscio, Andre Ismail, Christopher C Hull,
Christopher Liu

\begin{abstract}
Aim-To determine the long term visual and refractive results, and stability and complications of primary polypseudophakia using poly(methylmethacrylate) (PMMA) intraocular lenses (IOLs) for cataract surgery in hypermetropic eyes.

Methods-Prospective study of 15 short or hypermetropic eyes undergoing phacoemulsification with primary polypseudophakia with two PMMA IOLs implanted within the capsular bag.

Results-The spherical equivalent was reduced from a mean +4.87 (SD 3.00) dioptres (D) to $-0.12(1.40 \mathrm{D})$, and the deviation from the intended refraction was +0.005 (1.30) D, 23.6 (12.36) months post-implantation. The deviation from intended refraction was not statistically significant ( $\mathrm{p}=0.989$; paired $t$ test). Postoperative best corrected visual acuity (BCVA) was $6 / 12$ or better in all eyes without macular or optic nerve co-morbidity. Interlenticular opacification (ILO) in the form of peripheral Elschnig pearls was seen in four $(26.67 \%)$ eyes. A new type of ILO in the form of usually pigmented deposits in the central interface developed in five $(33.33 \%)$ eyes and resulted in the appearance of Newton's rings in three. None of the eyes with ILO had any loss of BCVA or hyperopic shift. Six $(40 \%)$ eyes were within $1 \mathrm{D}$ from the intended refraction and $14(93.33 \%)$ within $2 \mathrm{D}$. There was no statistically significant difference in the accuracy of the two intraocular lens calculation formulas used (SRK II and SRK/T).
\end{abstract}

Conclusion-Peripheral Elschnig pearltype ILO can occur as a late complication of primary in the bag implantation of two PMMA IOLs. A new type of ILO is described. Both types of ILO have not to date resulted in deterioration of visual acuity in our cohort. Use of appropriate biometry techniques and IOL calculation formulas may yield more accurate refractive results.

(Br f Ophthalmol 2001;85:1198-1202)

Polypseudophakia, also known as piggyback intraocular lens (IOL) implantation, was first described by Gayton in 1993, in a case of extreme hypermetropia in a nanophthalmic eye undergoing cataract surgery. As a +46 dioptres (D) IOL was not available, two IOLs were used with the first plano-convex lens implanted within the capsular bag (plano side facing anteriorly) and the second implanted in the ciliary sulcus (plano side facing posteriorly). ${ }^{1}$ Many surgeons have since used this technique for less extreme cases of hypermetropia, where a single high power IOL was out of the range or near the upper limit of power inventories. Implanting two IOLs in cases of high hypermetropia is preferable, because such systems provide better optical quality with less spherical aberration than a single high power IOL. ${ }^{2}$ Foldable piggyback IOLs have the added advantage of increased depth of focus due to mutual compression of the central optical zone of the implants. ${ }^{3-5}$ Primary polypseudophakia (primary implantation of two or more intraocular lenses at the time of cataract removal) has been used not only in short hypermetropic eyes after cataract surgery ${ }^{136-9}$ but also after refractive lensectomy, ${ }^{10}$ in myopic keratoconic eyes with cataract, ${ }^{11}$ and for the correction of paediatric aphakia. ${ }^{12}$ Secondary polypseudophakia $^{13}$ (implantation of a second intraocular lens at a later stage) has been used for the correction of refractive errors after cataract surgery, ${ }^{13-16}$ penetrating keratoplasty, ${ }^{14}{ }^{17}$ and refractive surgery in order to avoid the risks associated with intraocular lens exchange. ${ }^{14}$

\section{Materials and methods}

We carried out a prospective study of consecutive hypermetropic patients who underwent cataract surgery and primary implantation of two poly(methylmethacrylate) (PMMA) IOLs from January 1997 to October 1999. The purpose of our study was to determine the refractive results, the long term stability of the implants, and the complications of primary polypseudophakia. The inclusion criteria for the study were axial length (AL) of less than 21 $\mathrm{mm}$, or the requirement of an IOL of greater or equal to $+30 \mathrm{D}$ to achieve emmetropia. The AL was measured by applanation ultrasonography (Storz, CompuScan LT) with an ultrasound velocity of $1550 \mathrm{~m} / \mathrm{s}$. The IOL power was calculated using the SRK II formula in five eyes and the SRK/T formula in the other 10 eyes. The hospital was in transition to adopting the SRK-T formula as the standard formula, and two of the cases were treated in a private hospital. The intended postoperative refraction was emmetropic in all but two eyes where a slightly myopic refraction was required to avoid anisometropia.

All of the patients had nucleofractis phacoemulsification, with continuous curvilinear capsulorrhexis slightly smaller than the size of 
Table 1 Demographic details of the cohort of patients, their biometry, and their refractive and visual results

\begin{tabular}{|c|c|c|c|c|c|c|c|c|c|c|c|}
\hline No & Age & Sex & Preop refraction & $\begin{array}{l}\text { Preop spherical } \\
\text { equivalent }\end{array}$ & $\begin{array}{l}\text { Preop } \\
\text { BSCVA }\end{array}$ & $\begin{array}{l}\text { Axial } \\
\text { length }\end{array}$ & Formula & $\begin{array}{l}\text { Follow up } \\
\text { (months) }\end{array}$ & Postop refraction & $\begin{array}{l}\text { Postop spherical } \\
\text { equivalent }\end{array}$ & $\begin{array}{l}\text { Postop } \\
\text { BSCVA }\end{array}$ \\
\hline 1 & 60 & $M$ & $+9.50-2.00 \times 100$ & +8.5 & $6 / 18$ & 19.95 & $\mathrm{SRK} / \mathrm{II}$ & 47.00 & $+1.25-1.75 \times 140$ & +0.375 & $6 / 12$ \\
\hline 2 & 60 & M & $+8.50-2.00 \times 45$ & +7.5 & $6 / 18$ & 20.72 & $\mathrm{SRK} / \mathrm{T}$ & 44.00 & $+2.25-1.50 \times 40$ & +1.5 & $6 / 12$ \\
\hline 3 & 84 & $\mathrm{~F}$ & $+7.75-1.25 \times 75$ & +7.125 & $\mathrm{HM}$ & 19.44 & $\mathrm{SRK} / \mathrm{T}$ & 31.00 & $+0.25-2.75 \times 108$ & -1.125 & HM \\
\hline 4 & 84 & $\mathrm{~F}$ & $+7.25-0.75 \times 75$ & +6.875 & HM & 20.27 & SRK/T & 29.00 & $-0.00-0.50 \times 162$ & -1.25 & HM \\
\hline 5 & 84 & $\mathrm{~F}$ & $+5.75-1.50 \times 80$ & +5 & $6 / 18$ & 21.12 & SRK/II & 41.00 & $+2.75-2.75 \times 96$ & +1.375 & $6 / 18$ \\
\hline 6 & 70 & $\mathrm{~F}$ & $+7.50-3.00 \times 105$ & +6 & $6 / 12$ & 20.73 & SRK/II & 29.00 & $+3.50-3.00 \times 122$ & +2 & $2 / 60$ \\
\hline 7 & 91 & $\mathrm{~F}$ & $+9.00-2.00 \times 70$ & +8 & $6 / 18$ & 19.23 & SRK/II & 20.00 & $+2.00-2.00 \times 90$ & +1 & $6 / 12$ \\
\hline 8 & 82 & $\mathrm{~F}$ & Plano & 0 & $6 / 18$ & 20.83 & SRK/II & 13.00 & $-1.25-1.25 \times 95$ & -1.875 & $6 / 5$ \\
\hline 9 & 83 & $\mathrm{~F}$ & $+3.50-0.50 \times 60$ & +3.25 & $6 / 12$ & 20.70 & SRK/T & 13.00 & $-0.50-0.75 \times 90$ & -0.875 & $6 / 12$ \\
\hline 10 & 83 & $\mathrm{~F}$ & $+2.75-0.25 \times 30$ & +2.625 & $6 / 12$ & 20.85 & $\mathrm{SRK} / \mathrm{T}$ & 13.00 & $-0.50-2.50 \times 90$ & -1.75 & $6 / 9$ \\
\hline 11 & 94 & $\mathrm{~F}$ & Plano & 0 & $\mathrm{HM}$ & 20.43 & $\mathrm{SRK} / \mathrm{T}$ & 11.00 & $-1.00-2.50 \times 101$ & -2.25 & $\mathrm{CF}$ \\
\hline 12 & 72 & $\mathrm{~F}$ & $+2.00-1.50 \times 35$ & +1.25 & $6 / 18+1$ & 21.00 & $\mathrm{SRK} / \mathrm{T}$ & 17.00 & $-0.25 \mathrm{sph}$ & -0.25 & $6 / 9+3$ \\
\hline 13 & 71 & $\mathrm{~F}$ & $+9.25-1.00 \times 75$ & +8.75 & $6 / 12$ & 20.27 & $\mathrm{SRK} / \mathrm{T}$ & 16.00 & $+3.25-3.00 \times 63$ & +1.75 & $6 / 12$ \\
\hline 14 & 46 & $\mathrm{~F}$ & $+4.50-2.25 \times 150$ & +3.375 & $\mathrm{HM}$ & 20.59 & $\mathrm{SRK} / \mathrm{T}$ & 15.00 & $+0.50-1.50 \times 20$ & -0.25 & HM \\
\hline 15 & 46 & $\mathrm{~F}$ & $+5.50-1.50 \times 10$ & +4.75 & PL & 20.43 & $\mathrm{SRK} / \mathrm{T}$ & 15.00 & $+0.75-1.75 \times 110$ & -0.125 & HM \\
\hline
\end{tabular}

the IOL optic, through a $6 \mathrm{~mm}$ scleral tunnel incision. After aspiration of the cortical material, the capsular bag and the anterior chamber were inflated with a viscoelastic (hydroxypropyl methylcellulose) and the first biconvex one piece PMMA lens (Storz P359UV) was implanted in the bag. The second IOL of the same model was also implanted in the bag, after more viscoelastic was injected in the capsular bag. Occasionally, sodium hyaluronate including the high molecular weight version had to be used before implantation of the second IOL, especially when the anterior chamber was very shallow. The haptics of the two implants were dialled into parallel positions. ${ }^{89}$ The IOLs were of equal power where possible or near equal in the rest of the cases. The cohort of the patients was last examined between December 2000 and January 2001, so as to achieve a minimal follow up period of 12 months. Three patients had died and two were unable to attend because of poor health. The latest available data were used for these five patients.

Standard techniques were used for statistical analysis of data and surgically induced astigmatism was assessed with the vector approach described by Naeser and Hjordtal. ${ }^{18}$

\section{Results}

PATIENT CHARACTERISTICS

Fifteen eyes of 10 patients were included (two eyes of one male and 13 eyes of nine female patients). The follow up period was 23.6 (SD 12.36) months (range 11-47). The age of our patients at the time of surgery was 74 (15.13) years (range 46-94). The preoperative best corrected visual acuity (BCVA) was 6/12 in four eyes, 6/18 in six eyes, hand movements (HM) in four eyes, and perception of light (PL) in one eye. Ten eyes suffered from other ocular pathologies: seven eyes suffered from age related macular degeneration (ARMD) of various severity, four eyes from angle closure glaucoma (one eye had a previous attack of acute angle closure glaucoma and three eyes suffered chronic angle closure glaucoma), two eyes of a patient with Laurence Moon syndrome suffered from retinitis pigmentosa and one eye was amblyopic due to hyperopic anisometropia.

The preoperative spherical equivalent was +4.87 (3.00) D (range 0-+8.75 D) and the AL $20.44(0.54) \mathrm{mm}$ (range 19.23-21.12). The
IOL power required for emmetropia was +31.34 (1.71) D (range +28.42-+34.07), whereas the composite IOL power used was +31.47 (1.66) D (range +28.5-+35).

\section{COMPLICATIONS}

There were no operative complications.

VISUAL RESULTS

At the last study follow up visit, the Snellen BCVA in the eight eyes without severe macular or optic nerve pathology was $6 / 5$ in one eye, $6 / 9$ in two eyes, and 6/12 in five eyes, while from the other seven, one eye achieved $6 / 18$, one $2 / 60$, one counting fingers, and four eyes hand movements. On the whole $53.33 \%$ of the eyes achieved vision $6 / 12$ or better but by excluding the seven eyes with severe macular or optic nerve co-morbidity, $100 \%$ of the eyes achieved $6 / 12$ or better vision. The visual acuity improved in eight eyes $(53.33 \%)$, remained unchanged in six $(40 \%)$ due to concurrent ocular pathology, and deteriorated in one $(6.67 \%)$ due to progression of pre-existing exudative ARMD. No eye has lost vision because of the surgery.

\section{REFRACTIVE RESULTS}

The postoperative spherical equivalent was $-0.12(1.40) \mathrm{D}$ (range -2.25 to +2.00 ) .

The average induced astigmatism, calculated using a vector analysis, ${ }^{18}$ was $0.57 \mathrm{D}$ at 32 degrees. This was astigmatically neutral since it is not statistically significant ( $\mathrm{p}=0.182$; Hotelling's $\mathrm{T}^{2}$ test).

The vector analysis further revealed that surgery induced an effective against the rule astigmatism component of $-0.24 \mathrm{D}$ and that this was not statistically significant $(\mathrm{p}=0.536$; one sample $t$ test). The 135 degrees meridian was flattened by $-0.52 \mathrm{D}$ compared with the 45 degrees meridian although again this was not statistically significant ( $\mathrm{p}=0.077$; one sample $t$ test).

The deviation from the intended refraction was +0.005 (1.30) D (range -1.73-+2.06 D), which again is not statistically significant difference ( $\mathrm{p}=0.989$; paired $t$ test). Six eyes $(40 \%)$ were within $1.00 \mathrm{D}$ from the intended refraction and 14 eyes (93.33\%) were within $2.00 \mathrm{D}$. In the five eyes where the SRK II formula was used for the calculation of the IOL power, the postoperative deviation from the 

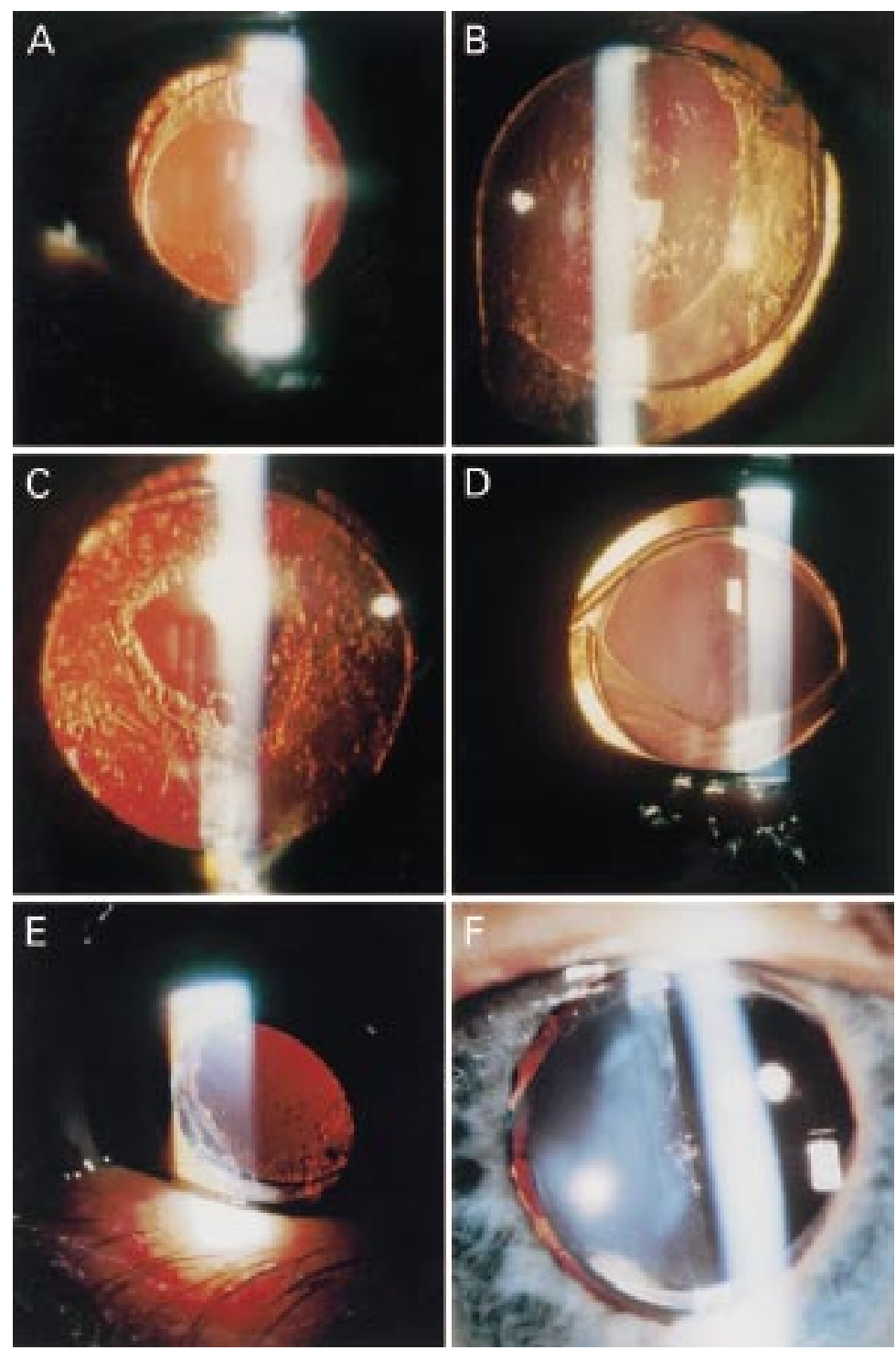

Figure 1 (A) Slit lamp photograph of case 9 demonstrating mild anterior capsular fibrosis. (B) Slit lamp photograph of case 3 demonstrating posterior capsular thickening. Despite an eccentric anterior capsulorrhexis, the two intraocular lenses remain well centred and the two sets of haptics aligned. (C) Slit lamp photograph of case 1 demonstrating a small YAG laser posterior capsulotomy in an otherwise opacified posterior capsule. (D) Slit lamp photograph of case 14 demonstrating an eccentric anterior capsulorrhexis and well centred optics and parallel haptics. (E) Slit lamp photograph of case 1 showing peripheral interlenticular opacities in the form of Elschnig pearls. (F) Slit lamp photograph of case 15 showing pigmented clumps near the visual axis in the interlenticular space. We have not been able to photograph the associated Newton rings satisfactorily.

intended refraction was $+0.76(1.28) \mathrm{D}$ (range $-1.375-+2.06)$, whereas in the 10 eyes where the SRK/T formula was used the deviation was -0.37 (1.19) D (range $-1.73-+1.70$ ). However this difference was not statistically significant ( $p=0.113$; Mann-Whitney test). In the SRK II group two eyes (40\%) were within 1.00 $\mathrm{D}$ from the intended refraction and four $(80 \%)$ within $2.00 \mathrm{D}$, whereas in the SRK/T group four eyes $(40 \%)$ were within $1.00 \mathrm{D}$ from the intended refraction and $10(100 \%)$ were within $2.00 \mathrm{D}$.

POSTOPERATIVE CAPSULAR CHANGES

The anterior capsule remained clear in nine eyes and became mildly fibrosed in six eyes (Fig 1A), but there was no clinical evidence of anterior capsular contraction in any of the eyes.

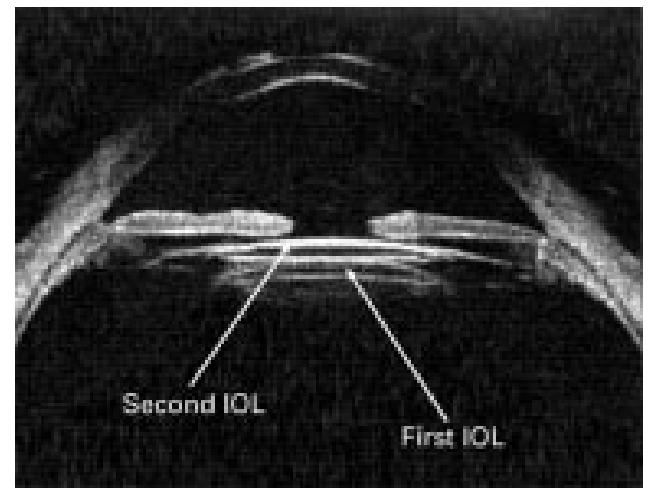

Figure 2 Montage of ultrasound biomicroscopy of the anterior segment of case 1 showing two intraocular lenses within the capsular bag.

The posterior capsule remained clear in five eyes and opacified in 10 eyes necessitating neodymium:YAG (Nd:YAG) laser posterior capsulotomy in seven eyes (Fig 1B and C). In the other three eyes the opacification was not sufficiently significant to justify capsulotomy.

INTRAOCULAR LENS STABILITY

On slit lamp examination through a maximally dilated pupil, all sets of haptics remained parallel within the capsular bag (Fig $1 \mathrm{~B}$ and D). However in five eyes part of the anterior and posterior lens optics were not covered by the anterior capsule, due to eccentricity of the capsulorrhexis (Fig 1B and D). There was no biomicroscopic evidence of tilt or decentration of the implants in any of the eyes (Fig 2).

\section{INTERLENTICULAR OPACIFICATION}

Interlenticular opacification (ILO) in the form of Elschnig pearls (Fig 1E) was noticed in the very peripheral interlenticular space in four $(26.67 \%)$ eyes. In two of them the ILO were first noticed almost 4 years postoperatively ( 47 and 44 months), whereas in the other two between the first and the second year. In three eyes the Elschnig pearl-type material extended less than 2 clock hours and in one eye about 12 clock hours in this space. In all cases the opacification was peripheral and extended less than $1 \mathrm{~mm}$ from the optic edge into the interlenticular space. None of the affected cases had the anterior capsulorrhexis edge resting outside the anterior optical surface of the anterior IOL. We have also observed another type of ILO in five eyes: precipitate-like deposits in the central interface (Fig 1F), which were brown pigmented in four eyes and non-pigmented in one. These were surrounded by Newton's rings in three eyes, which had the appearance of concentric light and dark zones. We first noticed their appearance 2 weeks after the operation in one eye of a patient with retinitis pigmentosa. The patient developed similar deposits in her other eye first noted at her 15 month postoperative visit. There was no evidence of intraocular inflammation in either eye. These deposits have remained unchanged since the time of their first appearance. In the other three eyes interface precipitates were observed 3, 29, and 41 months postoperatively. Four out of the five patients with the interface 
precipitates had eccentric capsulorrhexes outside the optic of the anterior lens for more than 5 clock hours. Although these interface opacities were situated near the visual axis they were not sufficiently extensive to reduce vision.

\section{Discussion}

The introduction of polypseudophakia by Gayton provided a solution to limitation of IOL power inventories, as the upper limit of most IOL models is $+30 \mathrm{D}$. An IOL with power of greater than $+40 \mathrm{D}$ would have very steep radii and would behave more like a sphere, resulting in increased spherical aberration, reduced modulation transfer function, and compromised resolution. ${ }^{2}$ Piggyback optical systems induce less spherical aberration and provide superior image quality when the optical centres of the two IOLs are aligned, than a single high power IOL of the same power. $^{2}$

IOL power calculation in short hyperopic eyes is a challenging problem. Accurate measurements of AL in these eyes are of great importance, since even small errors are magnified in inverse proportion to AL. A study performed by Holladay revealed that about $20 \%$ of the short hyperopic eyes have a short anterior segment, while the other $80 \%$ have normal anterior segment length. ${ }^{19}$ This diversity leads to refractive surprises in a number of short eyes, as empirical and most theoretical formulas assume a proportionately short anterior segment. Immersion biometry ${ }^{20}$ or the more recent partial coherence interferometry ${ }^{22}$ have been recommended as they avoid corneal indentation, which results in shorter AL measurements.

The empirical, and most regression calculation, formulas are not good at predicting the IOL power in short hyperopic eyes having primary piggyback IOLs for various reasons. ${ }^{36}{ }^{16}$ Current third generation formulas (Holladay and SRK/T) are superior to older formulas (Hoffer, SRK, and SRK II) for extremely short eyes, ${ }^{6}$ with the Holladay formula being more accurate than SRK/T. ${ }^{9}$ The Holladay II formula, which incorporates measurements of white to white corneal diameter, anterior chamber depth, lens thickness, takes into account the change in the position of the posterior IOL induced by the piggyback IOLs and predicts more accurately the required IOL power. It has very good predictability with almost $90 \%$ of the eyes within $1 \mathrm{D}$ from intended refraction. ${ }^{20}$ The Lloyd Gills regression formula with modifiers gives as good results as Holladay II but it creates more undercorrections and overcorrections. ${ }^{20}$

In our cohort SRK/T did not yield significantly more accurate results than the older SRK II formula. Neither of them was satisfactorily accurate.

Unlike the first reported case ${ }^{1}$ most surgeons have been implanting both primary piggyback IOLs in the bag until recently.5692324 We implanted all sets of IOLs in the capsular bag with the haptics parallel one set to another. All IOLs in our patients remained within the capsular bag with no evidence of IOL rotation (unlike other reported cases ${ }^{8}$ ), decentration, or tilt on biomicroscopy. ${ }^{25}$ Although part of the optics migrated in front of the anterior capsulorrhexis in five cases this was due to eccentricity of the capsulorrhexis.

Unlike the low PCO rate of primary AcrySof piggyback IOLs, ${ }^{23}$ we had high incidence of PCO in our cohort (10 out of 15 eyes, seven of which required Nd:YAG capsulotomy). This may be due to the use of PMMA IOLs as PCO is a material and shape dependent complication.

A visually significant complication occurring usually 6 months to 2 years post-implantation in primary piggyback IOLs is $\mathrm{ILO}^{24}$ (also known as interpseudophakos opacification $(\mathrm{IPO})^{26}$ and red rock syndrome ${ }^{27}$ ), which may lead to hyperopic shift, ${ }^{516}$ and loss of BCVA. ${ }^{23}$ The aetiology of ILO is primarily linked to a small size of capsulorrhexis resting on the anterior IOL surface. ${ }^{523} 24$ Various types of ILO have been described already ${ }^{23}$ including the Elschnig pearl-type observed in our cohort. Their appearance in our patients fits with the stage I of the clinical classification suggested by Stasiuk ${ }^{27}$ where scattered Elschnig pearls are seen in the periphery of the interface without any significant loss of BCVA or hyperopic shift.

We have observed a hitherto unreported type of ILO. This has the appearance of pigmented pseudophakic precipitates seen on single IOLs. Four of the five eyes with these interface precipitates had capsulorrhexes outside the anterior optical surface. Having a capsulorrhexis larger than the size of the optic for at least 1 clock hour does not prevent the appearance of this type of ILO, unlike previously described types of ILO $^{24}$ It is possible that these are formed by inflammatory cells trapped within the interface, with little aqueous circulation in this area. Although the precipitates are close to the visual axes in most cases, they have not caused any reduction of vision or change in refraction (hyperopic change).

These opacities seem to be different from the fine brownish grey dust-like material observed by Shugar ${ }^{23}$ which obscure the entire visual axis; they are discrete clumps close to each other occupying a small area. The presence of Newton's rings ${ }^{3}$ seen around the precipitates in three eyes signify a very small gap of $3 \mu \mathrm{m}$ or less between the two IOLs. The time of appearance of these clumps are variable, ranging from 2 weeks to 41 months postoperatively.

Unlike the general belief that Elschnig pearltype ILO develop between 6 months to 2 years, we noted the appearance of peripheral interface pearls after 3 years in two of our cases (44 and 47 months). We would therefore recommend indefinite follow up of patients with piggyback IOLs.

ILO is reportedly nearly twice as common in eyes with primary in the bag AcrySof than with PMMA $^{25} 28$ and has not yet been reported with primary or secondary piggyback IOLs in the ciliary sulcus. ${ }^{24}$ In our cohort we observed Elschnig pearl-type ILO in $26.67 \%$ of the eyes, which is comparable to the incidence of $22 \%$ found by Gayton and Apple in PMMA IOLs. ${ }^{28}$ 
In conclusion, primary polypseudophakia with two PMMA IOLs implanted in the capsular bag is linked to interlenticular opacification in the form of Elschnig pearls or interface precipitates. This may occur as a late complication even 3 years post-implantation. Therefore caution is required for the use of PMMA IOLs in the capsular bag as the long term outcome is not yet determined. In view of the early stages of ILO in our cohort we will continue monitoring the patients at regular intervals. The refractive results can be optimised by adoption of immersion or partial coherence interferometry biometry and careful selection of newer generation IOL power calculation formulas.

1 Gayton JL, Sanders VN. Implanting two posterior chamber intraocular lenses in a case of microphthalmos. $\mathcal{F}$ Cataract Refract Surg 1993;19:776-7.

2 Hull CC, Liu CSC, Sciscio A. Image quality in polypseudophakia for extremely short eyes. Br f Ophthalmol 1999;83 656-63.

3 Findl O, Menapace R, Rainer G, et al. Contact zone of piggyback acrylic intraocular lenses. F Cataract Refract Surg 1999;25:860-2.

4 Findl O, Menapace R. Piggyback intraocular lenses. 7 Cataract Refract Surg 2000;26:308-9.

5 Shugar JK, Schwartz T. Interpseudophakos Elschnig pearls associated with late hyperopic shift: a complication of pigassociated with late hyperopic shift: a complication of piggyback posterior chamber intraocular
Cataract Refract Surg 1999;25:863-7.

6 Holladay JT, Gills JP Leidlein J, et al. Achieving emmetropia in extremely short eyes with two piggyback posterior chamber intraocular lenses. Ophthalmology 1996;103:1118-23.

7 Mejia LF. Piggyback posterior chamber multifocal intraocular lenses in anisometropia. I Cataract Refract Surg 1999;25:1682-4.

8 Masket S. Piggyback intraocular lens implantation. 7 Cataract Refract Surg 1998;24:569-70.

9 Shugar JK, Lewis C, Lee A. Implantation of multiple foldable acrylic posterior chamber lenses in the capsular bag for high hyperopia. 7 Cataract Refract Surg 1996;22 1368-72.

10 Kolahdouz-Isfahani AH, Rostamian K, Wallace D, et al. Clear lens extraction with intraocular lens implantation for hyperopia. ₹ Refract Surg 1999;15:316-23

11 Gills JP. Piggyback minus-power lens implantation in Gills JP. Piggyback minus-power lens implantation

12 Mittelviefhaus $\mathrm{H}$. Piggyback intraocular lens with exchangeable optic. I Cataract Refract Surg 1996;22:676-81.
13 Liu C, Sciscio A, Hull C. Indications and technique for secondary polypseudophakia. [ARVO Abstract]. Invest Ophondary polypseudophakia. [ARVO Abstract]. Invest
thalmol Vis Sci 1999;40:(S) 455. (Abstract no 1547)

14 Gayton JL, Sanders V, Van Der Karr M, et al. Piggybacking intraocular implants to correct pseudophakic refractive error. Ophthalmology 1999;106:56-9.

15 Gills JP, Fenzl RE. Minus-power intraocular lenses to correct refractive errors in myopic pseudophakia. $\mathcal{F}$ Cataract Refract Surg 1999;25:1205-8.

16 Perrone DM. Modified intraocular lens power formula in polypseudophakia. I Cataract Refract Surg 1996;22:1392-3.

17 Gayton JL. Secondary implantation of a double intraocular lens after penetrating keratoplasty. 7 Cataract Refract Surg 1998;24:281-2.

18 Naeser K, Hjortdal JO. Bivariate analysis of surgically induced regular astigmatism. Mathematical analysis and graphical display. Ophthalmic Physiol Opt 1999;19:50-61.

19 Holladay JT. Achieving emmetropia in extremely short eyes. Presentation at the Annual Meeting of the American AcadPresentation at the Annual Meeting of the American Acad-

20 Fenzl RE, Gills JP, Cherchio M. Refractive and visual outcome of hyperopic cataract cases operated on before outcome of hyperopic cataract cases operated on before thalmology 1998;105:1759-64.

21 Shammas HJ. A comparison of immersion and contact techniques for axial length measurement. Am Intra-Ocular Implant Soc F 1984;10:444-7.

22 Drexler W, Findl O, Menapace R, et al. Partial coherence interferometry: a novel approach to biometry in cataract surgery. Am f Ophthalmol 1998;126:524-34.

23 Shugar JK, Keeler S. Interpseudophakos intraocular lens surface opacification as a late complication of piggyback crylic posterior chamber lens implantation. $\mathscr{f}$ Cataract Refract Surg 2000;26:448-55.

24 Gayton JL, Apple DJ, Peng O, et al. Interlenticular opacification: clinicopathological correlation of a complicaopacification: clinicopathological correlation of a complica-
tion of posterior chamber piggyback intraocular lenses. 7 Cataract Refract Surg 2000;26:330-6.

25 Hull CC, Liu CSC, Sciscio A. Degradation of retinal image quality due to tilt and decentration errors in polypseudohakia [ARVO abstract]. Invest Ophthalmol Vis Sci 1999;40:(S)296. (Abstract no 1568).

26 Shugar JK. Interpseudophakos opacification. Presentation at the Annual Meeting of the American Academy of Ophthalmology, Orlando, Florida, USA, October 1999

27 Stasiuk R. Red rock syndrome: interlenticular opacification with piggyback IOL implantation. Presentation at the Australian Society of Cataract and Refractive Surgeons Meeting, Ayers Rock, Australia, July 1999.

28 Gayton JL, Apple DJ. Refractive stability and long-term interlenticular membrane formation of piggyback intraocular implants. Presentation at the Annual Meeting of the American Academy of Ophthalmology, Orlando, Florida, USA, October 1999 .

29 Roy F. Membrane formation in primary piggyback intraocular lenses. Presentation at the American Society of Cataract and Refractive Surgeons Symposium on Cataract, IOL and Refractive Surgery, Boston, MA, USA, May 2000. 